\title{
Effects of 12 months of continuous positive airway pressure therapy on cognitive function, sleep, mood, and health-related quality of life in a peritoneal dialysis patient with obstructive sleep apnea
}

\author{
Kyoung Sook Park ${ }^{1}$, Jung Hyun Chang ${ }^{2}$, Ea Wha Kang ${ }^{1}$ \\ 'Division of Nephrology, Department of Internal Medicine, National Health Insurance Service Ilsan Hospital, Goyang, Korea \\ ${ }^{2}$ Department of Otorhinolaryngology, National Health Insurance Service Ilsan Hospital, Goyang, Korea
}

This report describes the case of a hypertensive 51-year-old male with a 3-year history of peritoneal dialysis. We followed the patient through his diagnosis of severe obstructive sleep apnea (OSA) and treatment with continuous positive airway pressure (CPAP). Therapeutic use of CPAP led to the improvement of not only sleep-related problems, but also cognitive function and quality of life. To our knowledge, this is the first paper describing the benefits of longterm CPAP treatment in an OSA patient undergoing dialysis. This case report emphasizes the need for the proactive diagnosis and treatment of OSA in end-stage renal disease patients to improve patient-centered healthcare.

Keywords: Continuous positive airway pressure, Obstructive sleep apnea, Peritoneal dialysis

\section{Introduction}

Obstructive sleep apnea (OSA) is a disorder characterized by recurrent arousal from sleep and intermittent hypoxemia; it is common in patients diagnosed with chronic kidney disease (CKD) and affects up to $70 \%$ of CKD patients [1]. Upper airway obstruction is distinguished by breathing cessations of at least 10 seconds accompanied by oxygen desaturation of $4 \%$ or greater, which occur along with inspiratory efforts during sleep. OSA is an important but often underdiagnosed and undertreated

Received December 1, 2017; Revised December 29, 2017;

Accepted January 8, 2018

Correspondence: Ea Wha Kang

Division of Nephrology, Department of Internal Medicine, National Health Insurance Service Ilsan Hospital, 100 Ilsan-ro, Ilsandong-gu, Goyang 10444, Korea. E-mail: eawha@nhimc.or.kr

ORCID: http://orcid.org/oooo-0003-0126-8132

Copyright (C) 2018 by The Korean Society of Nephrology

(c) This is an open-access article distributed under the terms of the Creative Commons Attribution Non-Commercial License (http://creativecommons.org/licenses/bync-nd/4.0/), which permits unrestricted non-commercial use, distribution, and reproduction in any medium, provided the original work is properly cited. sleep condition in the CKD patient population. OSA is associated with impaired health-related quality of life (HRQOL), excessive daytime sleepiness (EDS), depressed mood, reduced functional capacities, cognitive impairment, and hypertension, as well as increased risk of cardiovascular disease and higher mortality in the CKD and dialysis populations [2-5].

Continuous positive airway pressure (CPAP) therapy is widely accepted as the most effective therapy for OSA. In patients suffering from moderate to severe OSA, CPAP can improve cognitive function, daytime sleepiness, subjective sleep quality, HR-QOL, and mood [6,7]. However, poor compliance has been documented to lead to suboptimal treatment.

While there is one report about the acute beneficial effects of CPAP therapy on OSA in dialysis patients [8], there are no published reports on the effects of long-term CPAP therapy on cognition, daytime sleepiness, subjective sleep quality, mood, and HR-QOL in dialysis patients with OSA.

Here, we report improved daytime sleepiness, subjec- 
tive sleep quality, HR-QOL, and cognitive function after 12 months of CPAP therapy in a continuous ambulatory peritoneal dialysis (PD) patient with severe OSA.

\section{Case report}

A 51-year-old male with a body mass index of $25.2 \mathrm{~kg} / \mathrm{m}^{2}$ and neck circumference of $37 \mathrm{~cm}$, longstanding history of hypertension and chronic glomerulonephritis, non-smoker, and 3-year history of PD was assessed pretreatment and after 12 months of CPAP therapy. He was married and educated for 16 years. He was currently unemployed, but had a previous income over 3,500 US dollars a month. He had no previous history of cardiovascular disease. He was treated with four two-liter exchanges per day, using standard dialysates that contained glucose, and his $\mathrm{Kt} / \mathrm{V}$ was 1.98. His urine volume was $0.8 \mathrm{~L} /$ day, and residual renal function was $2.98 \mathrm{~mL} / \mathrm{min} / 1.73 \mathrm{~m}^{2}$. His volume status was measured with a Body Composition Monitor (BCM; Fresenius Medical Care, Bad Homburg, Germany), and was determined to have normohydration status (TBW; 38.0 L and overhydration; $-0.6 \mathrm{~L}$ ).

The patient participated in a prospective cohort study that investigated memory, sleep, depression, and quality of life in patients undergoing PD. He took three antihypertensive medications, including a calcium channel blocker, angiotensin receptor blocker, and diuretics, and his blood pressure was 148/90 $\mathrm{mmHg}$ at baseline. Baseline laboratory tests presented the following values: hemoglobin, $11.2 \mathrm{~g} / \mathrm{dL}$; serum albumin, $3.1 \mathrm{~g} / \mathrm{dL}$; serum creatinine, $14.3 \mathrm{mg} / \mathrm{dL}$; total cholesterol, $103 \mathrm{mg} / \mathrm{dL}$; calcium, $9.5 \mathrm{mg} / \mathrm{dL}$; phosphorous, $4.5 \mathrm{mg} / \mathrm{dL}$, parathyroid hormone, $276.9 \mathrm{pg} / \mathrm{mL}$. The patient completed a standardized health interview; questionnaires focused on daytime sleepiness (the Epworth Sleepiness Scale, ESS) and sleep quality (the Pittsburgh Sleep Quality Index, PSQI), depression (the Patient Health Questionnaire-9, PHQ-9), and quality of life (the Kidney Disease Quality of Life-36, KDQOL-36); a questionnaire on anthropometric measurements (height, weight, neck circumference); and a neuropsychological examination (the Korean version of Alzheimer's Disease Assessment Scale, ADAS-K; and Montreal Cognition Assessment, MoCA). Unattended in-home polysomnography (PSG) was performed at habitual sleep time using an ambulatory Watch-PAT 100 (Itamar Medical Ltd., Caesarea, Israel). The Watch-PAT test revealed the patient's apnea hypopnea index (AHI) to be 58.8 events per hour, with an $87 \% \mathrm{SaO}_{2}$ nadir. $\mathrm{He}$ was referred to the otorhinolaryngology clinic for further evaluation of his OSA. Upon physical examination, the patient was found to have nasal septal deviation to the right and turbinate hypertrophy. An elongated uvula and a low, flaccid soft palate were noted, which could cause airway collapse. Comprehensive overnight PSG, which was conducted in the hospital with a Neuvo PSG Trolley (Compumedics Ltd., Abbotsford, VIC, Australia), showed severe sleep apnea with an AHI of 50.7 events per hour and a $78 \% \mathrm{SaO}_{2}$ nadir (Table 1). Respiratory events were close to obstructive, with few central apneas. CPAP with a mean pressure of $4.8 \mathrm{~cm} \mathrm{H}_{2} \mathrm{O}$ curtailed the obstructive respiratory events to an AHI of 2.5 events per hour. The patient admitted to nightly use of the CPAP for an average of six to seven hours. Follow-up tests for cognition, sleep, mood, and HR-QOL were performed after 12 months of CPAP treatment.

Twelve months after CPAP therapy, the patient reported feeling more refreshed in the morning and a considerable improvement in memory. His daytime vigilance greatly improved with treatment, and his post-treatment ESS score was 6, which was lower than the pre-treatment score of 15 . He showed improved cognitive function (especially in constructional praxis), executive function, verbal delayed recall memory, and orientation in the

Table 1. Data from patient polysomnography

\begin{tabular}{lc}
\hline \multicolumn{1}{c}{ Variable } & Data \\
\hline TST (min) & 425 \\
Sleep efficiency (\%) & 91.6 \\
Stage 1 (\%TST) & 13.4 \\
Stage 2 (\%TST) & 62.8 \\
REM (\%TST) & 11.4 \\
AHI (event/hr) & 50.7 \\
Obstructive apnea & 86 \\
Central apnea & 0 \\
Mixed apnea & 0 \\
Hypopnea & 273 \\
Nocturnal hypoxemia (\%)* & 9.7 \\
SaO & Nadir (\%) \\
PLMI (/hr) & 78 \\
\hline
\end{tabular}

AHI, apnea-hypopnea index; PLMI, periodic leg movement index; REM, rapid eye movement; TST, total sleep time.

$\star \mathrm{SpO}_{2}<90 \%$ for $\geq 3 \%$ TST. 
Table 2. Changes in cognitive function, sleep, mood, and quality of life before and after 12 months of CPAP therapy

\begin{tabular}{lcc}
\hline \multicolumn{1}{c}{ Domain } & Baseline & $\begin{array}{c}\text { After 12 months of } \\
\text { CPAP therapy }\end{array}$ \\
\hline Cognitive function & & 4 \\
ADAS-K, total score & 7 & 0 \\
$\quad$ Constructional praxis & 1 & 0 \\
$\quad$ Orientation & 1 & 1 \\
$\quad$ Word recognition & 25 & 29 \\
MoCA, total score & & \\
Sleep & 15 & 6 \\
Daytime sleepiness (ESS) & 6 & 6 \\
Sleep quality (PSQI) & & 8 \\
Mood & 10 & \\
PHQ-9 & & 93.0 \\
Quality of life (KDQOL-36) & 60.0 & 87.5 \\
Cognitive function & 62.5 & 70.0 \\
Sleep & 50.0 & 95.0 \\
Overall health & 85.0 & 65.0 \\
Physical functioning & 45.0 & 68.0 \\
Fatigue & 64.0 & 73.0 \\
Emotional well-being & 53.0 & 100.0 \\
Quality of social interaction & 87.5 & \\
Social function & 515 & \\
\hline
\end{tabular}

ADAS-K, the Korean version of Alzheimer's Disease Assessment Scale; CPAP, continuous positive airway pressure; ESS, Epworth Sleepiness Scale; KDQOL-36, Kidney Disease Quality of Life-36; MoCA, Montreal Cognition Assessment; PHQ9, Patient Health Questionnaire-9; PSQI, Pittsburgh Sleep Quality Index.

ADAS-K and MoCA test. His mood test results indicated that the depression symptoms had improved, although there was little difference. He also reported a great improvement of HR-QOL in terms of cognition, sleep, overall health, physical function, and fatigue, as well as improvements in social interactions, in the KDQOL-36 questionnaire. Table 2 details the test results for cognitive function, sleep, mood, and HR-QOL before and after the 12 months of CPAP treatment (Figs. 1, 2).

\section{Discussion}

In this case report, clinically important manifestations of severe OSA were assessed in a PD patient prior to and 12 months after treatment with a nasally applied CPAP treatment. CPAP treatment proved to be highly successful; this approach reduced AHI to normal levels, and also improved daytime sleepiness, as well as the HRQOL domains of cognition, sleep, overall health, physical

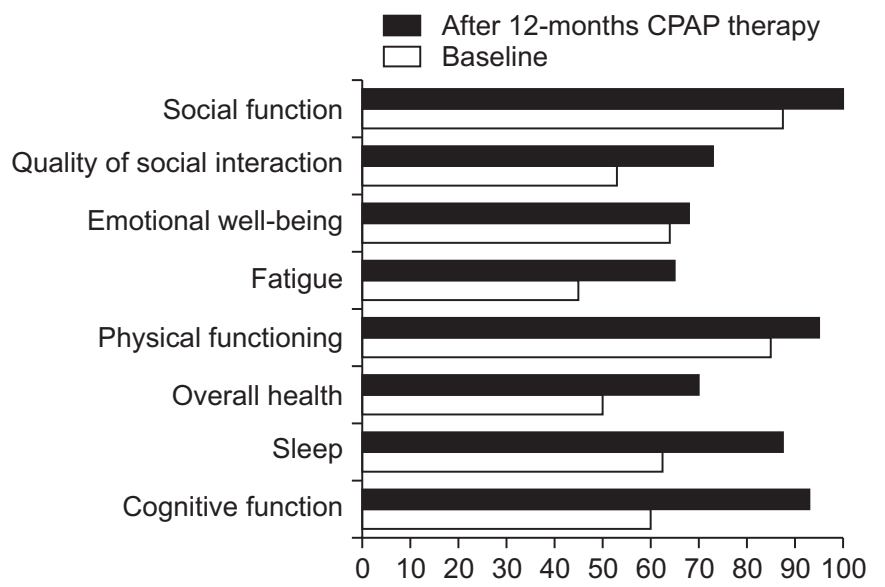

Figure 1. Health-related quality of life after continuous positive airway pressure (CPAP) therapy.

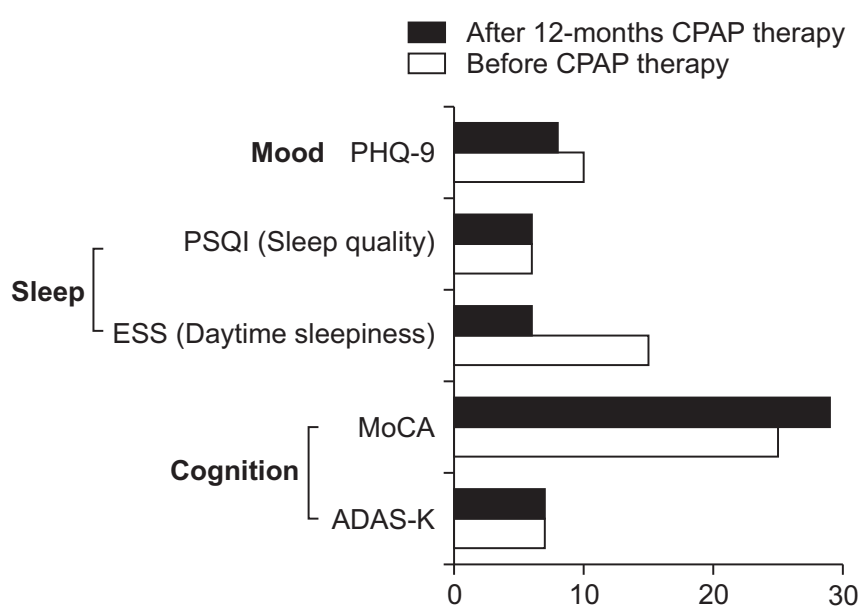

Figure 2. Daytime vigilance, mood, cognition after continuous positive airway pressure (CPAP) therapy.

ADAS-K, the Korean version of Alzheimer's Disease Assessment Scale; ESS, Epworth Sleepiness Scale; MoCA, Montreal Cognition Assessment; PHQ-9, Patient Health Questionnaire-9; PSQI, Pittsburgh Sleep Quality Index.

function, fatigue, and social interaction. Interestingly, the patient reported feeling more refreshed in the morning with a considerable enhancement in memory. This corresponds with previous findings that varying periods of CPAP therapy improved memory function in patients with OSA [7].

Although OSA is highly prevalent and can severely impact outcomes in end-stage renal disease (ESRD) patients, it remains significantly underdiagnosed and undertreated by nephrologists for various reasons, such as lack of awareness and screening tools, low PSG accessibility, and poor patient compliance [1]. The Watch- 
PAT 100 test, which was used as a screening test in this study, is a simple approach that can help overcome these limitations. The test is less expensive than others, can be conducted on a portable monitor, and can easily be selfadministered at home. The American Academy of Sleep Medicine recommended using portable monitors to assess OSA in patients with high pretest potential for the disease [9]. Additional testing is recommended if OSA is suspected, particularly with the aim of early diagnosis in ESRD populations. The diagnostic strategy includes a questionnaire to evaluate sleep quality and EDS, along with a physical examination and objective sleep testing.

OSA is associated with low HR-QOL [2], which not only includes patients' perception of their own well-being, but is also a major prognostic marker in patients undergoing dialysis. In two meta-analyses of OSA patients, CPAP improved physical ability, energy/vitality, and general well-being, as measured by SF- 36 [10,11]. OSA is also associated with cognitive impairments, especially of verbal memory, in patients with advanced CKD [4]. However, one trial reported improvements in verbal memory function following 3 months of CPAP therapy [7]. Cognitive impairment in CKD not only increases the risk of mortality, but may also lead to poor HR-QOL and suboptimal medical care because of difficulties in following the caregiver's recommendations regarding dietary restrictions, pharmacologic therapy, dialysis initiation and maintenance, and transplantation [12]. Despite the multiple known benefits of CPAP therapy in patients with OSA, there is a paucity of data on the effects of CPAP treatment in ESRD patients. Pressman et al [8] studied the acute impact of CPAP on eight ESRD patients with sleep disordered breathing, successfully administering CPAP to six of the patients, and found a significant improvement in nocturnal oxygenation and daytime alertness. Our case report shows that long-term CPAP therapy can be tolerated well, in spite of concerns about patient compliance. Additional factors related to renal failure, such as fluid overload and azotemia, can also contribute to the increased propensity to OSA observed in these populations. AHI, which is a severity index parameter of OSA, has been reported to be more effectively alleviated by nocturnal PD or HD than by continuous ambulatory PD or conventional HD due to greater net ultrafiltration during sleep and better uremic clearance [13-15]. After controlling for volume and uremia, CPAP could be con- sidered primary therapy for OSA in ESRD patients.

Our study provides an additional, useful option to address the risk factors for HR-QOL, cognitive impairment, and cardiovascular morbidity in CKD and dialysis patients with OSA. Additional, larger studies of CPAP therapy in ESRD patients with OSA are necessary to further investigate whether CPAP therapy contributes to reducing HR-QOL, morbidity, and mortality in these high-risk populations.

\section{Conflicts of interest}

All authors have no conflicts of interest to declare.

\section{References}

[1] Dharia SM, Brown LK, Unruh ML. Recognition and treatment of obstructive sleep apnea. Semin Dial 26:273-277, 2013

[2] Sanner BM, Tepel M, Esser M, et al. Sleep-related breathing disorders impair quality of life in haemodialysis recipients. Nephrol Dial Transplant 17:1260-1265, 2002

[3] Unruh ML, Sanders MH, Redline S, et al. Subjective and objective sleep quality in patients on conventional thriceweekly hemodialysis: comparison with matched controls from the sleep heart health study. Am J Kidney Dis 52:305313, 2008

[4] Kang EW, Abdel-Kader K, Yabes J, Glover K, Unruh M. Association of sleep-disordered breathing with cognitive dysfunction in CKD stages 4-5. Am J Kidney Dis 60:949-958, 2012

[5] Zoccali C, Mallamaci F, Tripepi G. Nocturnal hypoxemia predicts incident cardiovascular complications in dialysis patients. J Am Soc Nephrol 13:729-733, 2002

[6] Antic NA, Catcheside P, Buchan C, et al. The effect of CPAP in normalizing daytime sleepiness, quality of life, and neurocognitive function in patients with moderate to severe OSA. Sleep 34:111-119, 2011

[7] Zimmerman ME, Arnedt JT, Stanchina M, Millman RP, Aloia MS. Normalization of memory performance and positive airway pressure adherence in memory-impaired patients with obstructive sleep apnea. Chest 130:1772-1778, 2006

[8] Pressman MR, Benz RL, Schleifer CR, Peterson DD. Sleep disordered breathing in ESRD: acute beneficial effects of treatment with nasal continuous positive airway pressure. 
Kidney Int 43:1134-1139, 1993

[9] Epstein LJ, Kristo D, Strollo PJ Jr, et al. Clinical guideline for the evaluation, management and long-term care of obstructive sleep apnea in adults. J Clin Sleep Med 5:263-276, 2009

[10] Jing J, Huang T, Cui W, Shen H. Effect on quality of life of continuous positive airway pressure in patients with obstructive sleep apnea syndrome: a meta-analysis. Lung 186:131-144, 2008

[11] Giles TL, Lasserson TJ, Smith BH, White J, Wright J, Cates CJ. Continuous positive airways pressure for obstructive sleep apnoea in adults. Cochrane Database Syst Rev (3):CD001106, 2006

[12] Roumelioti ME, Buysse DJ, Sanders MH, Strollo P, Newman
AB, Unruh ML. Sleep-disordered breathing and excessive daytime sleepiness in chronic kidney disease and hemodialysis. Clin J Am Soc Nephrol 6:986-994, 2011

[13] Tang SC, Lam B, Lai AS, et al. Improvement in sleep apnea during nocturnal peritoneal dialysis is associated with reduced airway congestion and better uremic clearance. Clin J Am Soc Nephrol 4:410-418, 2009

[14] Hanly PJ, Pierratos A. Improvement of sleep apnea in patients with chronic renal failure who undergo nocturnal hemodialysis. N Engl J Med 344:102-107, 2001

[15] Chan CT, Hanly P, Gabor J, Picton P, Pierratos A, Floras JS. Impact of nocturnal hemodialysis on the variability of heart rate and duration of hypoxemia during sleep. Kidney Int 65:661-665, 2004 